\title{
La cabane éclatée. Morcellement des objets immobiliers apparentés à l'art brut
}

The exploded cabin. Splitting up structures related to art brut

\section{Roberta Trapani}

\section{OpenEdition}

\section{Journals}

Édition électronique

URL : http://journals.openedition.org/cel/493

DOI : $10.4000 /$ cel. 493

ISSN : 2262-208X

Éditeur

École du Louvre

\section{Édition imprimée}

Date de publication : 1 avril 2014

\section{Référence électronique}

Roberta Trapani, «La cabane éclatée. Morcellement des objets immobiliers apparentés à l'art brut », Les Cahiers de l'École du Louvre [En ligne], 4 | 2014, mis en ligne le 01 avril 2014, consulté le 21 avril 2019. URL : http://journals.openedition.org/cel/493 ; DOI : 10.4000/cel.493

\section{(c) $(1) \odot$}

Les Cahiers de l'École du Louvre sont mis à disposition selon les termes de la licence Creative Commons Attribution - Pas d'Utilisation Commerciale - Pas de Modification 4.0 International. 


\section{Cahiers de l'École du Louvre}

recherches en histoire de l'art, histoire des civilisations archéologie, anthropologie et muséologie

\section{Numéro 4. Avril 2014}

\section{La cabane éclatée.}

Morcellement des objets immobiliers

apparentés à l'art brut

Roberta Trapani

Article disponible en ligne à l'adresse :

http://www.ecoledulouvre/cahiers-de-l'ecole-du-louvre/numero4avril2014/Trapani.pdf

Pour citer cet article :

Roberta Trapani, « La cabane éclatée. Morcellement des objets immobiliers apparentés à l'art brut », Cahiers de l'Ecole du Louvre, recherches en histoire de l'art, histoire des civilisations, archéologie, anthropologie et muséologie [en ligne] n 4, avril 2014, p. 87 à 95 .

\section{cc) creative \\ B.: $\ominus \$$}

Gécoled at Lowre

Cet article est mis à disposition selon les termes de la Licence Creative Commons Attribution - Pas d'utilisation commerciale - Pas de modification 3.0 non transposé. 


\section{Cahiers de l'École du Louvre recherches en histoire de l'art, histoire des civilisations archéologie, anthropologie et muséologie \\ Numéro 4. Avril 2014}

Sommaire

Éditorial

p. 1

\section{Étude}

Le musée de Sculpture comparée au prisme de la collection de cartes postales éditées par les frères Neurdein (1904-1915)

Dominique Jarrassé et Emmanuelle Polack

p. $2-20$

Dossier:

Les modalités de la collecte : rapt, troc, marché, fouilles, don...

Et leur impact sur l'objet

Dans collection, il y a collecte...

Introduction de Dominique Jarrassé.

p. $21-23$

Entrer en collection. Pour une ethnographie des gestes et des techniques de collecte

Julien Bondaz

p. $24-32$

De la meilleure façon de constituer une collection. Le cas des émaux «byzantins » de Mikhaill Botkine

Aglaé Achechova.

Edme Antoine Durand (1768-1835) : un bâtisseur de collections

Louise Detrez p. $45-55$

Réunir une documentation pour l'Art Brut : les prospections de Dubuffet dans l'immédiat après-guerre au regard du modèle ethnographique Baptiste Brun p. $56-66$

Travailler sur des objets en faisant se confronter les points de vue : regards croisés sur le langage fou : l'exemple des écrits bruts

Vincent Capt p. $67-75$

D’un regard-pilote à l'écart. L'impact de Dubuffet sur les collectes de l'association L'Aracine

Déborah Couette. p. $76-86$

La cabane éclatée. Morcellement des objets immobiliers apparentés à l'art brut

Roberta Trapani .p. $87-95$ 


\section{La cabane éclatée. Morcellement des objets immobiliers apparentés à l'art brut}

\section{Roberta Trapani}

Depuis les années 1930, les objets immobiliers que nous avons tendance aujourd'hui à apparenter à l'art brut ont attiré l'intérêt de plusieurs acteurs du monde de la culture et des arts ${ }^{1}$. À l'origine, ces productions n'ont aucune prétention artistique et d'objets délaissés ou négligés, ils deviennent au cours de leur existence des objets qui intéressent au plus haut point les musées. Cette transformation est largement due à la réception et à la perception changeante qu'ils subissent au cours du temps ${ }^{2}$. Dans ce processus de reconnaissance, le moment de la collecte est essentiel. Cependant, avant de nous y consacrer, nous allons nous focaliser sur le point initial de leur trajectoire sociale : leur fabrication, à savoir leur entrée dans la société. Appréhender la logique dans laquelle leur matérialisation s'inscrit permet de définir un ensemble cohérent d'objets et de montrer comment notre appréhension change au moment où ils entrent dans des collections.

\section{Récolter, remployer, représenter : de l'objet abject à l'objet animé}

Il s'agit, à l'origine, de très modestes lieux de vie qui, à travers des opérations pratiques et symboliques, subissent une altération : d'univers domestiques, ils sont transformés en univers esthétiques répondant à de nouvelles exigences de la part de leurs habitants. Les auteurs de cette transformation matérielle, et du déplacement de sens qui s'en suit, sont les habitants mêmes. Ce sont des non professionnels de l'art et de l'architecture, ils appartiennent majoritairement aux classes populaires et à la frange inférieure des classes moyennes. Ils interviennent dans un espace souvent situé entre la clôture de leur jardin et la façade de leur maison. Les modalités d'intervention sont en général la réalisation d'artefacts (sculptures, peintures, assemblages) qu'ils déclinent sur la moindre surface disponible, débordant, parfois, jusqu’à envahir l'espace public.

Il est intéressant de remarquer que ces objets immobiliers, qui seront soumis dans la deuxième partie de leur vie à plusieurs types de collecte, naissent eux-mêmes d'une quête et d'une récolte soigneuses et acharnées. Le manque de moyens économiques, qui conditionne habituellement l'habitat populaire, pousse ces habitants à négliger toute autre occupation pour se consacrer d'abord à l'art du glanage, puis à celui du bricolage. Dans leur environnement immédiat, ils récupèrent les matériaux qui, au gré du hasard, les séduisent. Ce qui les fait marcher, ce qui oriente leurs pas, ce sont des reliques de sens, et parfois des déchets : des objets naturels ou des résidus industriels, trouvés sur des chantiers ou dans des décharges. Pendant un intervalle de temps qui dure généralement des décennies et correspond souvent au temps de réalisation d'un site, des éléments matériels de catégories différentes sont glanés, stockés, classés et, enfin, recyclés ou remployés. À travers de multiples manipulations, ces détritus cessent d'être des objets abjects pour être mis en œuvre. Un glissement de sens se produit alors : des

1. Pour une bibliographie récente sur le sujet en France, voir Marc Decimo, Les Jardins de l'art brut, Dijon, Les presses du réel, 2007 ; Marielle Magliozzi, Art brut, architectures marginales : un art du bricolage, Paris, L'Harmattan, 2008 ; Bruno Montpied, Éloge des jardins anarchiques, Paris, L'Insomniaque, 2011.

2. Nous faisons référence ici à la "cultural biography of things", méthode préconisée par Igor Kopytoff et Arjun Appadurai dans Arjun Appadurai, The Social Life of Things. Commodities in Cultural Perspective, Cambridge University Press, Cambridge, 1986. 
choses immondes se changent en héritages aux multiples possibilités plastiques et expressives ${ }^{3}$.

À l’image de "la coquille de l'escargot " évoquée par Bachelard, " la maison qui grandit à la mesure de son hôte " ${ }^{4}$, ces sites évoluent suivant le rythme de vie de leurs auteurs, qui les modifient sans cesse. La lenteur et la répétitivité de leur activité technique, sa pratique routinière, n'est cependant pas, chez eux, synonyme d'ennui, mais plutôt de plaisir, car loin d'être une activité abrutissante, elle favorise au contraire l'acquisition de compétences, l'imagination et l'innovation ${ }^{5}$. Sans un projet strictement défini à l'avance, en procédant par suppositions, expériences, essais hasardeux et erreurs, en répétant et entremêlant des sujets et des modules expressifs et en accumulant des formes jusqu'à la saturation de l'espace, ces " habitants-paysagistes ${ }^{6}$ » mettent en œuvre une capacité à penser et à inventer dans le faire. La conception de leurs travaux naît de l'exécution. Ils utilisent un outillage rudimentaire et jouent avec les différents matériaux et procédés, qu'ils inventent ou réinventent, en se référant parfois aux savoirs hérités de l'artisanat traditionnel. «Le "bourrage" spatial et le "bourrage" temporel s'appellent et se répondent mutuellement pour les producteurs d'environnements extraordinaires ", remarque le sociologue Jean-Pierre Martinon ${ }^{7}$; leur production « est rarement fixée une fois pour toutes, elle est, au contraire, en perpétuel renouvellement, ou en incessante et irrépressible prolifération ${ }^{8} »$. Un acte de ré-création, ludique, libre et gratuit est à l'origine d'ensembles structurés, colorés, kitsch et en même temps inventifs : des façades historiées, des clôtures ouvragées, des constructions ingénieuses, des bestiaires de ciment, des assemblages monumentaux. Dans ces lieux, la fonction sociale du "fabriquer " de l'Homo faber fait écho à celle du jeu propre à l'Homo ludens, modèle anthropologique décrit par Huizinga en $1938^{10}$, puis désigné par Constant comme l'habitant idéal de New Babylon, la ville situationniste ${ }^{11}$. C'est aux théâtres d'actions et situations évoqués par Constant que nous font penser, en effet, ces espaces où la création et l'exploration coïncident et où le temps utile, le temps de la consommation passive, se transforme en temps ludico-constructif. Pour les analyser, nous pourrions emprunter à Michel de Certeau sa notion d'espace : non pas un lieu géométrique et stable, mais un "croisement de mobiles" fonctionnant en " unité polyvalente de programmes conflictuels ou de proximités contractuelles $»^{12}$. Ces théâtres d'actions et situations sont en effet constamment " animé[s] par l'ensemble des mouvements qui s'y déploient ${ }^{13}$ » et dont l'habitant-paysagiste n'est pas le seul acteur.

3. Voir Laura Foulquier, «La métamorphose des pierres. Les remplois, entre rebut et souvenir », Cannibalismes disciplinaires. Quand I'histoire de l'art et l'anthropologie se rencontrent, dir. Thierry Dufrêne et Anne-Christine Taylor, Paris, Institut national d'histoire de l'art, Musée du quai Branly, 2009, pp. 335-345 ; Roberta Trapani, « Le jardin de Litnianski : une stratégie créative de résistance $\gg, 50^{\circ}$ Nord, n० 2, 2011, pp. 140-145.

4. Gaston Bachelard, La poétique de l'espace, Paris, PUF, 1957, p. 116.

5. Une réhabilitation du travail artisanal comme métier qui fait aussi bien appel à la tête qu'à la main, et une réévaluation du rôle de la routine dans le processus de création, dans Richard Sennett, Ce que sait la main. La culture de l'artisanat [2008], traduit de l'américain par PierreEmmanuel Dauzat, Paris, Albin Michel, 2010. L'auteur tient à remercier Eva di Stefano (Université de Palerme) pour cette suggestion bibliographique.

6. L'expression est inventée par l'architecte Bernard Lassus, à la suite d'une exploration menée en France de 1967 à 1974, voir Bernard Lassus : paysages quotidiens, de l'ambiance au démesurable, cat. d'exp. réalisé par le MNAM-CCI, Musée des arts décoratifs, Paris, 8 janvier-9 mars 1975, Paris, Centre de création industrielle, 1975 ; B. Lassus, « Les Habitants paysagistes », Jardins imaginaires, les habitants paysagistes, Paris, Presses de la Connaissance, 1977.

7. Jean-Pierre Martinon, «Systèmes de l'hétéroclite et de l'éphémère. Les habitants bricoleurs : leurs demeures et leurs jardins », Architecture et comportement : revue internationale et interdisciplinaire consacrée aux relations entre être humain et environnement construit, vol. 1 , $\mathrm{n}^{\circ} 2,1980 / 81$, p. 88

8. Ibid., p. 91 .

9. Pour une analyse de ce concept au XXe siècle, voir Henri Bergson, L'Évolution créatrice [1907], Paris, PUF, 1959 ; Hannah Arendt, The Human Condition, Chicago, University of Chicago Press, 1958.

10. Johan Huizinga, Homo Ludens, Paris, Gallimard, 1951.

11. Nieuwenhuys Constant, « New Babylon, une ville nomade », Cause commune, $\mathrm{n}^{\circ}$ spécial « Nomades et vagabonds », Paris, U.G.E., collection 10/18, 1975, pp. 202-230.

12. Michel de Certeau, L'invention du quotidien 1. Arts de faire, Paris, Gallimard, 1990, p. 173.

13. Idem, ibidem. 


\section{Lieux d'échange, terrains de jeu}

Si une pratique routinière et signifiante permet à l'habitant de réactiver dans l'isolement l'intelligence de sa propre main, la mise en place de ses créations dans un lieu spécifiquement livré à l'admiration et à la sanction du groupe répond à son désir de capter le regard de l'autre et de se confronter à celui-ci. Les habitants du site environnant, les promeneurs, sont appelés à l'observer à l'œuvre pendant qu'il collecte ses matériaux ou réalise ses productions, ils sont invités à participer à l'évolution du site et à se transmettre la légende d'une entreprise qui modifie non seulement un espace de vie personnel, mais aussi l'habitat collectif. Identitaires et relationnels à la fois, ces objets évoquent d'une part les réflexions du philosophe et naturaliste américain Henry David Thoreau, pour lequel bâtir sa propre maison est comme construire sa propre pensée ${ }^{14}$. Ils nous conduisent, d'autre part, à utiliser la notion de cabane, décrite par le philosophe français Gilles A. Tiberghien comme " une façon d'être non pas à l'abri du monde, mais hors de soi $»^{15}$. La façade, le jardin, les sculptures sont disposés selon des relations de coexistence par lesquelles le créateur construit et représente son identité ou met en scène l'identité dont il rêve, ses fantasmes conscients et inconscients, son image idéale. Mis en devanture, à la portée du regard venant de l'extérieur, ces "égo-musées ${ }^{16}$ " sont, d'une certaine manière, cédés volontairement à l'autre, ils font l'objet d'un don, cet acte qui, selon Jean Bazin, est en mesure de transformer même un objet " usuel et ordinaire » en une chose qui a une force, " une chose unique ${ }^{17}$. Selon Véronique Moulinié, « le système de l'échange, du don et du contre-don est [...] un puissant moteur pour cette production ${ }^{18}$ " que l'habitant offre métaphoriquement à la communauté et qui lui sert de support et de prétexte pour interagir avec le monde extérieur. Ces objets immobiliers « supposent, pour prendre sens, que s'établisse un dialogue entre le créateur et l'observateur. L'essentiel, leur raison d'être est sans doute à chercher dans ce discours qu' [ils] rendent possible ${ }^{19}$ " et qui symbolise, à notre avis, le contre-don auquel les auteurs aspirent. S'ils cèdent à la communauté leurs objets, ils demandent en contrepartie son regard et sa parole. Un échange symbolique a donc lieu dans ces sites. Il se fonde sur ce que Michel de Certeau appelle " récits d'espace ${ }^{20}$ ", des actions narratives par lesquels l'habitant explicite à l'observateur sa pratique organisatrice d'espace, permettant ainsi au système de signes qu'il invente d'être pratiqué, parcouru, expérimenté. Cette expérience relationnelle fortifie son identité, satisfaisant en même temps à son besoin d'entretenir et de raffermir les sentiments collectifs.

\section{Passage à l'art: le rôle des collectes photographiques}

Dépourvus du langage des arts, les habitants-bâtisseurs sont évasifs quand il s'agit de définir la raison d'être de leur pratique. Ils ne revendiquent pas pour leurs réalisations le qualificatif d'art ou d'œuvre, ni pour eux-mêmes celui d'artiste. "Ça n'a pas de définition ", affirme Fernand Châtelain (1899-1988), auteur d'un jardin de sculptures à Fyé, dans la Sarthe, interviewé vers 1980 par le sociologue Jean-Pierre Martinon dans le cadre d'une recherche sur les pratiques d'embellissement de la vie quotidienne. " "Ça n'a pas de définition", mais ça

14. Henry David Thoreau, Walden ou la Vie dans les bois, Paris, Gallimard, 1992, p. 47.

15. Gilles A. Tiberghien, Nature, Art, Paysage, Arles, Actes-Sud, 2001, p. 119. Objet anthropologique renvoyant en permanence à l'imaginaire et à des univers techniques, sociaux, culturels et symboliques, la cabane représente un outil précieux pour comprendre ces ensembles qui condensent savoir-faire et mode de vie.

16. Véronique Moulinié, «L'autre de I'art - Production "œuvrière" et égo-musée », communication inédite, colloque Art spontané, art brut, art psychopathologique, direction scientifique Benoît Decron, organisé par Galerie nationale du Jeu de Paume à l'occasion de l'exposition Chaissac, Studio-Théâtre de la Comédie Française (Carrousel du Louvre), Paris, 14 octobre 2000. http://halshs.archives-ouvertes.fr/halshs-00141941, consulté le 25 avril 2014.

17. Jean Bazin, «Essais », Des clous dans la Joconde : I'anthropologie autrement, Toulouse, Anacharsis, 2008, p. 554.

18. V. Moulinié, « Des "œuvriers" ordinaires. Lorsque l'ouvrier fait le/du beau... », Terrain, n 32, mars 1999

http://terrain.revues.org/2825, consulté le 25 avril 2014.

19. V. Moulinié, op. cit. note 16, p. 6.

20. Avec l'expression « récit d'espace », de Certeau décrit des récits qui parcourent et organisent des lieux, mais aussi l'espace construit par l'écriture même du récit. Voir M. de Certeau, op. cit. note 12 , pp. 170-191. 
existe et ça fait exister, réplique Martinon, cela marque le territoire en l'identifiant et cela donne un sens à l'existence $~^{21}$. C'est justement cette volonté de marquer le territoire et de capter le regard de l'autre qui détermine l'entrée de ces créateurs et de leurs productions quotidiennes modestes dans le monde de l'art. La pratique photographique est un instrument privilégié de ce processus d'artification ${ }^{22}$.

Figure 1:

Rothéneuf : I'ermite Adolphe Julien Fouré sculptant les rochers photographie en noir et blanc H. 0,11 ; L. $0,28 \mathrm{~m}$. cliché Germain fils - Saint-Malo

(c) Collection J.-M. Chesné

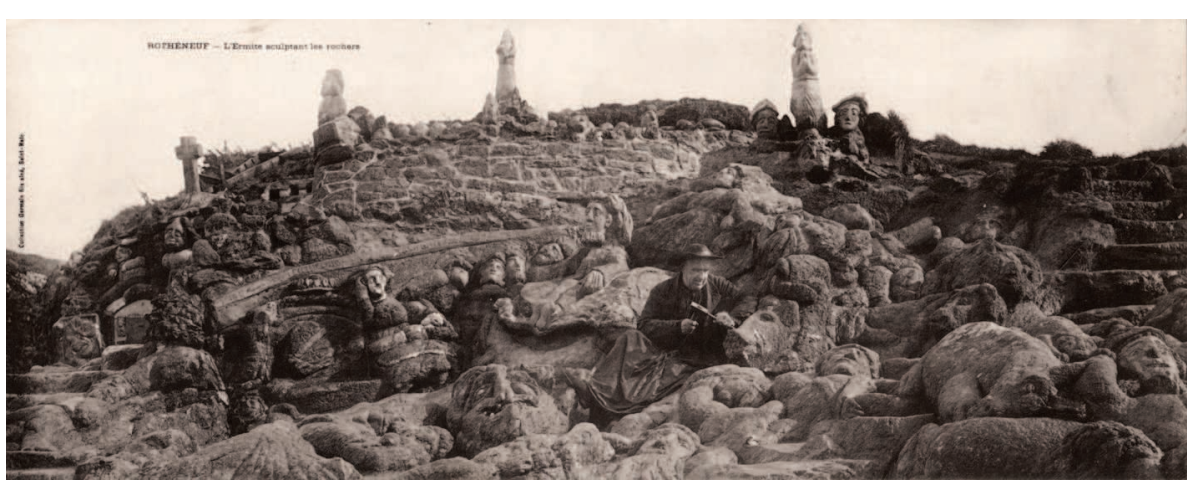

Avant d'avoir achevé son Palais idéal, le facteur Ferdinand Cheval (1836$1924)^{23}$ le fait photographier. Il choisit de se montrer avec affectation face à sa majestueuse construction ou avec ses outils rudimentaires. Il signe ainsi, métaphoriquement, sa construction. Quant à l'abbé Fouré (1839-1910), il authentifie par un tampon les cartes postales illustrées de photographies de ses Rochers sculptés ${ }^{24}$ de Rothéneuf (fig. 1). Ces reproductions, ainsi construites par leurs auteurs, commencent à circuler dans la presse locale, puis nationale, modifiant la réception des objets mêmes ${ }^{25}$. Considérés, à l'origine, comme des curiosités locales, voire comme l'expression de la dévotion ou de l'extravagance populaire, ils sont vite remarqués par des acteurs du monde de l'art et notamment par les surréalistes, qui ne tardent pas à les qualifier d'objets poétiques susceptibles d'effacer la frontière entre production extraordinaire et quotidienneté ordinaire. Cette reconnaissance artistique conduit à la protection éditoriale de certains sites, en premier lieu de l'œuvre architecturale de Cheval, révélée dans une revue d'art en $1929^{26}$. Pendant plusieurs années, la renommée ou mieux la sacralisation de ce Palais idéal occulte l'existence d'objets immobiliers similaires ${ }^{27}$. Pour assister à la parution, dans des revues d'art, d'articles dédiés à ces élaborations, il faut attendre les années d'après-guerre. Les premiers à prendre des images de ces sites et à les construire intellectuellement, en leur assignant une fonction identitaire et sociale, sont Robert Doisneau (1912-1994) et Gilles Ehrmann (1928-2005), photographes proches du surréalisme.

À compter des années d'après-guerre, les images que Doisneau rassemblait depuis le début des années 1940 commencent à circuler dans des revues spécialisées, souvent accompagnées d'écrits donnant lieu à des interprétations

21. J.-P. Martinon, op. cit. note 7, p. 85.

22. Une première réflexion sur la notion $d^{\prime}$ « artification » dans Roberta Shapiro, «Qu'est-ce que l'artification? », XVII Congrès de I'AISLF « L'individu social », Tours, juillet 2004.

http://hal.archives-ouvertes.fr/index.php?halsid=00003aep0r9t4dqlghl76504n1\&view_this_ doc $=$ halshs-00010486\&version $=2$, consulté le 25 avril 2014.

Voir : De l'artification. Enquêtes sur les passages à l'art, collectif dirigé par Nathalie Heinich et Roberta Shapiro, EHESS, février 2012.

23. Le Palais idéal est construit, de 1879 à 1912, à Hauterives (Drôme) par Ferdinand Cheval, facteur rural.

24. De fin 1894 à 1907, I'abbé Adolphe Julien Fouré sculpte de nombreuses figures dans des rochers granitiques surplombant la mer à Rothéneuf, en Bretagne.

25. Pour l'impact des cartes postales sur la reconnaissance des sites insolites, voir Jean-François Maurice (dir.), Gazogène, Cahors, $\mathrm{n}^{\circ} 24$, $\mathrm{n}^{\circ} 27$ et $\mathrm{n}^{\circ}$ hors-série « N'oubliez pas l'artiste ». Non datés, ces numéros révèlent l'importante collection de cartes postales de Jean-Michel Chesné.

26. « Le Surréalisme en 1929 », Variété, Bruxelles, n hors-série, juin 1929. Deux cartes postales du Palais idéal sont publiées dans la cinquième page d'illustrations (n. p.) suivant la p. 38.

27. Pour une étude du pouvoir de séduction exercé par le Palais idéal sur les surréalistes et pour une analyse des affinités de cette création avec le projet surréaliste, voir : « Le surréalisme sans I'architecture », Mélusine, n 29 dossier réuni par Henri Béhar et Emmanuel Rubio, Lausanne, L'Àge d'Homme, 2009. Dans ce collectif, voir surtout : Jean-Claude Blachère, « La maison abolie », pp. 37-49; Robert Ponge, Nara Machado, « De I'Art nouveau au Palais idéal : des architectures en marge de l'architecture », pp. 95-106. Dans cet esprit, voir aussi : Éric Le Roy, «Le facteur Cheval et les Surréalistes », cat. d'exp. Avec le facteur Cheval, Musée de la poste, Paris, 6 avril-1 ${ }^{\text {er }}$ septembre 2007, Musée de la poste, École nationale supérieure des beaux-arts, 2007, pp. 26-33. 
poétiques $^{28}$. Déplacés par l'image, ces sites quittent alors leur emplacement réel pour prendre place dans un livre, dans le studio d'un amateur, dans un espace d'exposition ou dans une collection. Le consensus produit par ces reproductions photographiques modifie leur statut : ils sont qualifiés d'œuvres, leurs auteurs de "bâtisseurs chimériques " ou d' "inspirés $»^{29}$. Et si les images qu'Ehrmann commence à rassembler vers 1956 sont dévoilées en 1962 dans l'ouvrage évocateur Les Inspirés et leurs demeures, préfacé par André Breton ${ }^{30}$, c'est dans Bizarre, revue influencée par le surréalisme, que Jean-Hugues Sainmont publie en 1955 le premier article de fond sur un habitant sui generis: Camille Renault (1866-1954), auteur d'un jardin de sculptures à Attigny ${ }^{31}$. Pâtissier et animateur de fêtes, Renault avait reconstruit deux fois sa maison à l'architecture ingénieuse, ainsi que son "Jardin des Surprises » où une nature artificielle hébergeait un carnaval de personnages pittoresques et familiers façonnés en ciment peint aux couleurs vives $^{32}$ (fig. 2). Il y avait mis en scène sa mythologie quotidienne, transformant ainsi son habitat en un espace d'expérimentation et de représentation. Si le texte de Sainmont présentait cet auteur comme " créateur du Monde » et figurait sa production environnementale sous forme d'un " cosmos " se manifestant " dans le vide préoriginel ", il n'était pas pour autant qu'un panégyrique enthousiaste. Teinté de poésie, il rassemblait et révélait aussi l'important travail documentaire mené par Sainmont au cours de nombreux voyages, ainsi que de nombreuses prises de vues réalisées par plusieurs opérateurs.

Figure 2 :
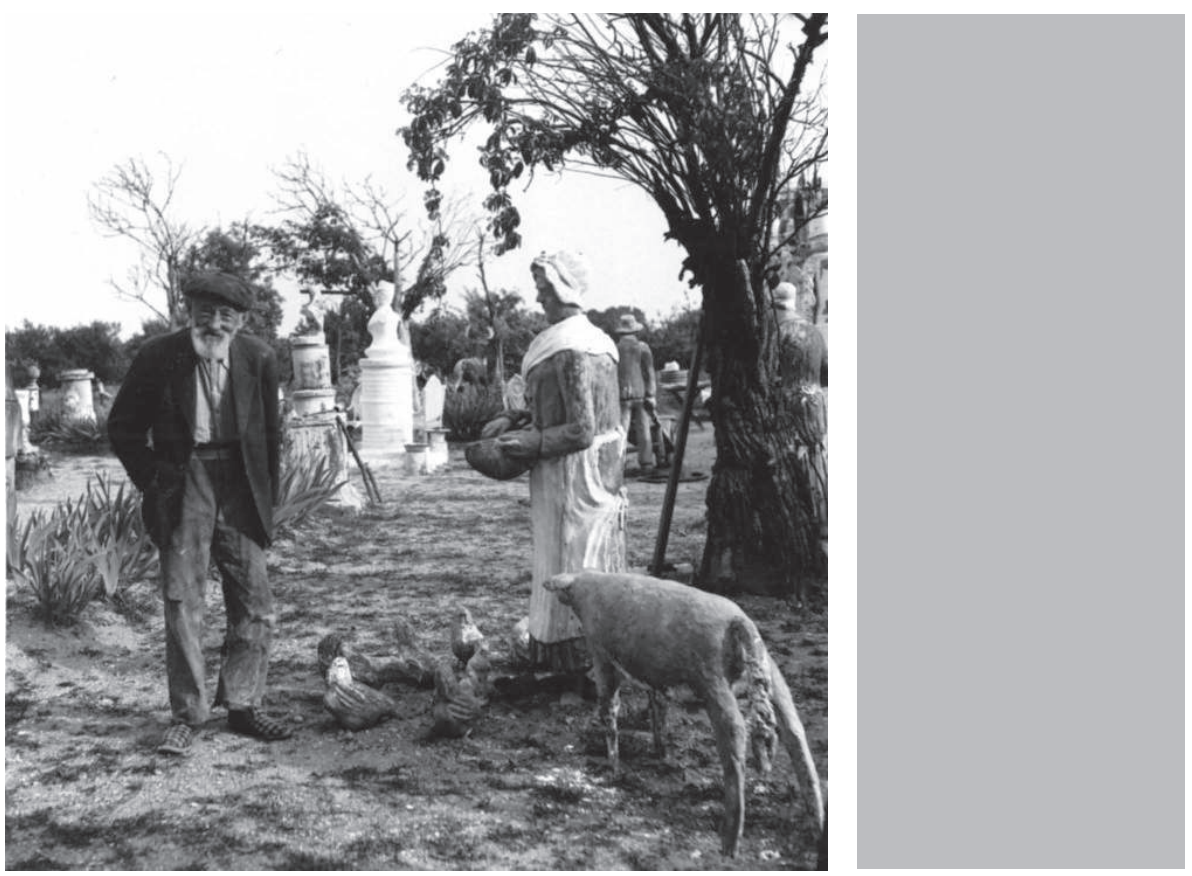

Ces collectes d'images et de documents ont le mérite d'avoir sauvé la mémoire de ce lieu intrinsèquement fragile du fait des techniques expérimentales avec lesquelles il avait été créé, et fragilisé ultérieurement par l'indifférence et les pillages qui suivent le décès de son auteur. En 1956, un an après la publication du texte de Sainmont et deux ans après la mort de Renault, Doisneau se rendra sur place et ce n'est pas la beauté du lieu qui le surprendra, mais sa ruine. Entretemps, le site avait en effet été " massacré par les gosses d'Attigny ", comme le déplore le photographe, qui ajoute : "Les générations montantes n'ont rien à foutre du passé. Ras-le-bol contracté avec l'enseignement scolaire. Je suis arrivé

28. Voir par exemple : Robert Giraud, «Étoiles noires de Paris : Frédéric Séron est le bon Dieu du paradis des animaux », Paris-Presse-I'Intransigeant, avril 1950 ; Id., « Château des assiettes cassées », Nouveau Fémina, septembre 1955, pp. 86-89.

29. Voir Charles Soubeyran, Les révoltés du merveilleux, avec photographies de Robert Doisneau et Gilles Ehrmann, Cognac, le Temps qu'il fait, 2004.

30. Gilles Ehrmann, Les Inspirés et leurs demeures, Paris, Le Temps, 1962.

31. Jean-Hugues Sainmont, «Camille Renault. Créateur du Monde », Bizarre, n², Paris, Le Minotaure, octobre 1955, pp. 2-18.

32. Ces sculptures étaient faites d'un bâti de treillage ou de tôles de jardin et comportaient à l'intérieur des objets de récupération : poêles, bidons, pièces de vélo, etc. 
trop tard, j'ai vu le charnier, l'herbe avait poussé et faisait disparaittre les statues décapitées $»^{33}$ (fig. 3).

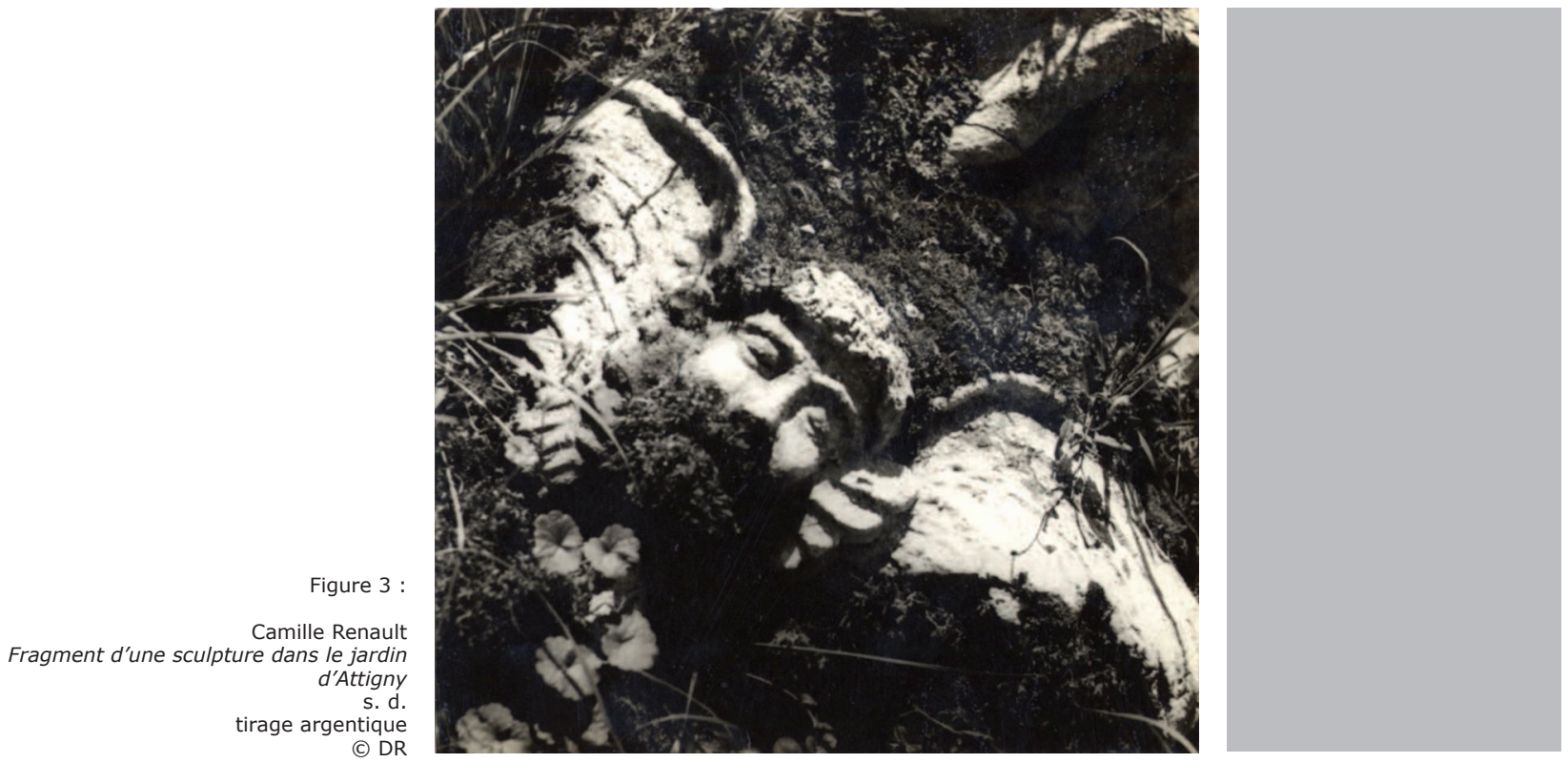

\section{Des ensembles, en morceaux: principe d'une carrière idéale?}

Dans les années 1960, grâce à la publication d'articles et de photographies et notamment au succès recueilli par l'ouvrage de Ehrmann ${ }^{34}$ (Prix Nadar, 1963), on assiste à une véritable vogue pour ces objets immobiliers et à une nouvelle étape de leur passage à l'art. Cette fois-ci, l'instrument privilégié en est le morcellement. Arrachés à leur contexte d'origine, recueillis parfois in extremis, des débris de sites attirent l'attention de collectionneurs et d'amateurs, en premier lieu de Jean Dubuffet, qui les intègre à sa collection. Transformés en objets mobiliers, ces fragments sont alors labellisés art brut et, subséquemment, les sites mêmes desquels ils proviennent sont annexés à cet art.

L'un des premiers sites qui subit cette nouvelle mutation de statut est celui de Camille Renault. Début 1960, Raymond Fleury, alors Satrape du Collège de Pataphysique, accomplit un acte de vandalisme tutélaire ${ }^{35}$ pour sauver ce qui restait du "Jardin des surprises " d'Attigny. Grâce à son intermédiaire, certaines pièces sont collectées par Jean Dubuffet ${ }^{36}$ (fig. 4) qui, les considérant " merveilleuses" au point qu'elles auraient pu stimuler son travail ${ }^{37}$, choisit de les présenter en 1967 dans l'exposition L'Art brut organisée aux Arts Décoratifs ${ }^{38}$. Lassimilation à l'art brut amène d'autres fragments du site de Renault à rejoindre d'autres collections privées apparentées à celle de Dubuffet. Vers 1985, Renée Jakovsky offre à Madeleine Lommel, présidente de L'Aracine, une tête sculptée de sa collection d'art naif, qui rejoint ainsi cette collection d'art brut ; en 2000, Bruno Decharme achète un balustre anthropomorphe provenant de la maison du sculpteur, objet puissant en soi, qu'il intègre à sa collection ABCD (art brut connaissance \& diffusion). Il faut cependant rappeler qu'en 1984 Dubuffet

33. Robert Doisneau cité dans C. Soubeyran, op. cit. note 29, p. 100.

34. G. Ehrmann, op. cit. note 30 .

35. Dans une lettre adressée à Jean Dubuffet le 21 août 1962, Raymond Fleury écrit : « À vous lire je regrette de n'avoir pas pu enlever, jusqu'aux moindres miettes, tout ce qui reste des $œ u v r e s$ de Camille Renault à Attigny. [...] je crois bien que je suis repéré. Au cours du dernier enlèvement, alors que nous venions de charger la fermière et qu'on se préparait à aller chercher une autre pièce, un voisin est venu avertir le propriétaire que nous avons vu accourir accompagné de ses trois chiens », Lausanne, archives de la Collection de l'Art Brut.

36. La Collection de I'Art Brut possède : Ange, ciment, H. 0,98 m (provenant de l'ensemble disparu de la Pietà); Fermière jetant du grain aux poules, ciment, H. 1,25 m ; Lion (fragment), ciment, H. 0,65 m ; Buste de fantaisie, béton armé, H. 0,66; L. 0,41 m.

37. Lettre de Jean Dubuffet à Roger Cornaille datée du 17 octobre 1962, Lausanne, archives de la Collection de l'Art Brut.

38. L'Art brut, cat. d'exp., Musée des arts décoratifs, 7 avril-5 juin 1967, texte de Jean Dubuffet, Paris, Compagnie de I'Art Brut \& Union Centrale des Arts Décoratifs, 1967, p. 97. 
était revenu sur ses positions, jusqu'à dire, à propos des sculptures de Renault, qu'elles " ne sont pas fortement inventives, en ce sens qu'elles ne s'écartent pas radicalement de l'art traditionnel ${ }^{39} \%$. Ce changement de position ne doit pas étonner. L'annexion de ces objets immobiliers à l'art brut est, en effet, toujours problématique. Faits pour être communiqués et « combinant en des proportions en chaque fois singulières, des éléments traditionnels, naïfs ou bruts ${ }^{40}$ ", ils sont souvent très différents des œuvres de l'art brut fantasmé par Dubuffet, à savoir des productions idéalement écartées de l'art culturel et d'autant plus intenses qu'elles ne sont pas faites dans un processus de communication. C'est pour cette raison qu'ils sont souvent considérés par la suite comme des objets " apparentés " à cet art.

Figure 4:
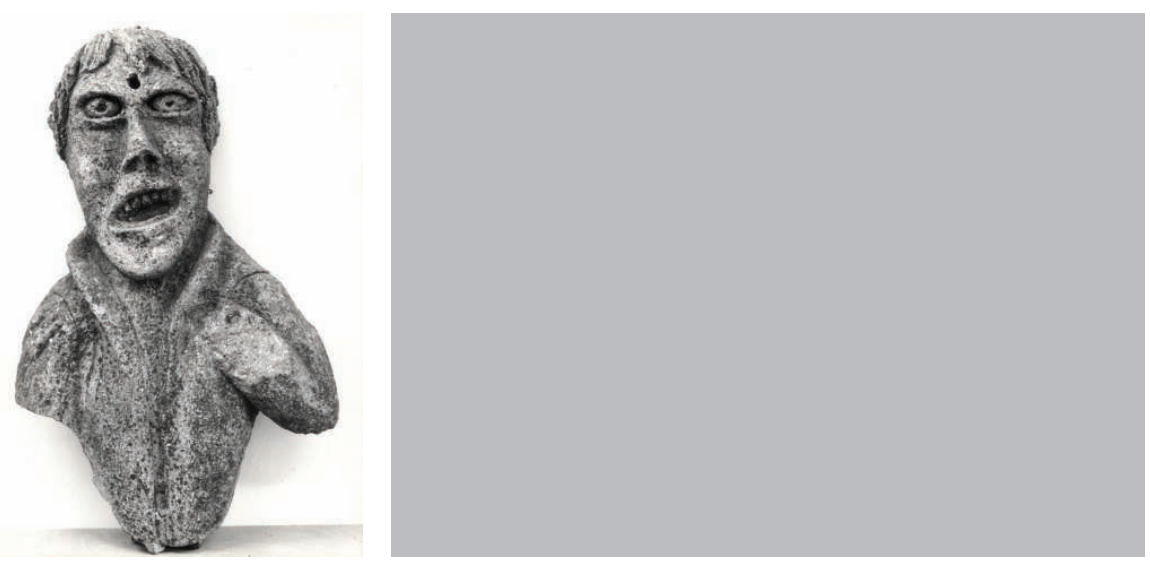

Le morcellement et la dispersion du jardin de Renault n'est pas un phénomène exceptionnel, un sort similaire attend beaucoup d'autres environnements irréguliers. La sauvegarde in situ de ces objets, susceptible, sinon de garder leur caractère évolutif et interactif, au moins de préserver leur intégralité et de les maintenir dans leur contexte d'origine, est en effet problématique et donc très rare. Étrangers au système de l'art, les habitants-paysagistes se préoccupent rarement de la conservation de leurs créations. Après leur décès, elles risquent de disparaittre pour des raisons diverses. On constate, par exemple, une faible résistance des objets aux intempéries. Réalisées avec des techniques improvisées et des matériaux inappropriés, ces créations se révèlent le plus souvent éphémères. De plus, nécessitant un entretien constant et étant parfois dénoncées comme des constructions illégales, elles suscitent des réactions contrastées chez les héritiers. La photographie, ainsi que la vidéo, peut représenter un moyen de pérenniser ces objets évolutifs et éphémères, en les changeant en objets d'étude. Et pourtant, il faut reconnaitre la partialité de cette pérennisation, car toute prise de vue ne propose jamais qu'une vérité fragmentaire.

Une altération similaire se produit quand, pour soustraire ces objets à l'oubli, on les fragmente. Contrairement aux collectes d'images, ces récoltes de morceaux se produisent avec difficulté du vivant des auteurs. Insoucieux de se voir consacrer un espace dans une collection, ceux-ci acceptent avec difficulté d'aliéner, par le don ou par le marché, leurs propres productions et de fragmenter ainsi leur " égo-musée ". Ces collectes se produisent alors, le plus souvent, après leur décès, quand les sites sont, non seulement, dépouillés de leur caractère performatif, processuel et relationnel, mais se voient aussi menacés de disparition. Intégré dans une collection, l'objet-fragment est alors conservé, mais pour ce faire, il doit perdre sa raison d'être première, qui était celle de faire sens dans un ensemble, un parcours, un récit. Dans un but paradoxal de conservation, le collectionneur de fragments participe activement à la disparition de l'objet tel qu'il était conçu

39. Lettre de Jean Dubuffet datée du 16 octobre 1984, dans Camille Renault : créateur d'univers, cat. d'exp. sous la direction d'Annie Bissarette et Pascal Sigoda, Charleville-Mézières, Lycée Chanzy, 12-20 avril 1985, Musée Rimbaud, 22 avril-5 mai 1985, Lycée Chanzy, CharlevilleMézières, 1985, p. 2.

40. Raymond Moulin, Les Singuliers de l'art, des inspirés aux habitants-paysagistes, cat. d'exp. sous la direction de Susanne Pagé, ARC 2, Musée d'art moderne de la ville de Paris, 19 janvier-5 mars 1978, Musée d'art moderne de la ville de Paris, 1978, n. p. 
par son auteur, car, de l'ensemble, il ne peut sauver que des reliques ${ }^{41}$. Le " récit d'espace » qu'était le site habité par son auteur éclate alors, donnant lieu à un exode de récits de mémoire. Mais si le morcellement d'un site se révèle souvent nécessaire, existe-il un moyen, autre que la sauvegarde in situ, pour retrouver le sens que le site avait du vivant de son auteur?

\section{Remise en contexte et retour de sens : la voie des conservateurs}

Dans l'exposition Camille Renault : créateur d'univers, organisée en 1985 par Pascal Sigoda, certains fragments du site de Renault, éparpillés dans des collections diverses, sont présentés dans un même espace ${ }^{42}$. Sortis des réserves et côtoyant d'autres créations de l'auteur, dont des machines et des tableaux, ainsi que des objets lui appartenant, ces fragments retrouvent une certaine unité, grâce surtout à l'importante documentation que Sigoda choisit de présenter - photographies d'archive, écrits, données sociologiques. L'effort de remise en contexte fait par Sigoda est, à notre avis, exemplaire. On en retrouve un écho dans les démarches accomplies depuis quelques années par le LaM de Villeneuve d'Ascq ${ }^{43}$, qui à l'étude de ces objets applique une perspective biographique. Emblématique en ce sens est le parcours du parc de totems en bois du menuisier Théo Wiesen (19061999), transplanté du bord d'un bois de Belgique aux salles d'exposition de ce musée. Alignées le long de la route avoisinant sa maison (fig. 5), certaines

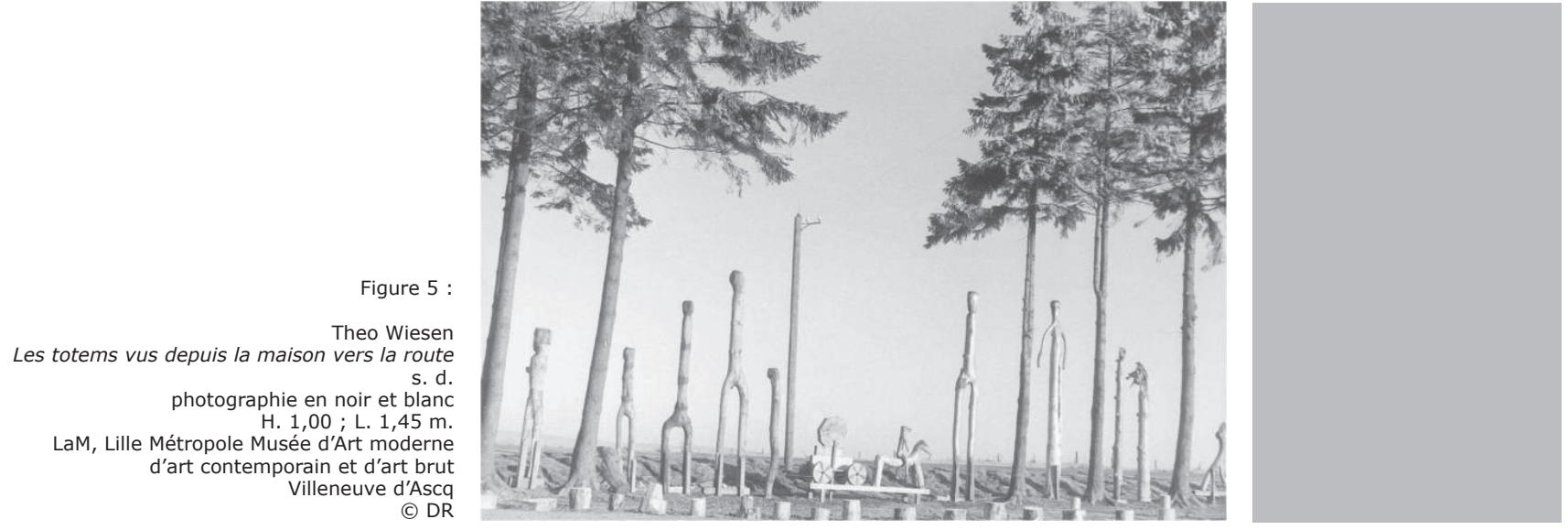

sculptures de Wiesen avaient été collectées par Claire Teller de L'Aracine dès 1988, puis en 1999, après le décès de l'auteur et par le biais d'une vente. Animés par la volonté de mettre en perspective ces sculptures énigmatiques, les conservateurs de la section art brut du LaM choisissent de réaliser des nouvelles collectes, de fragments, mais surtout de données. "Quand les oeuvres ont été collectées par les membres de L'Aracine, il n'y a pas eu de repérage, donc au début on ne savait pas du tout comment le site avait été constitué», affirme Savine Faupin, conservateur en chef en charge de l'art brut ${ }^{44}$. En s'inspirant de l'exploration systématique faite par Bernard Lassus ${ }^{45}$, le LaM compose alors une équipe. Larchitecte Aurélie Harnéquaux fait des repérages à partir de cartes topographiques et essaye de comprendre, à partir de photographies, l'emplacement des totems et des éléments de barrière. L'ethnologue Véronique Moulinié fait des enquêtes. Le photographe Philippe Bernard réalise des nouvelles prises de vue du site qui,

41. Pour des questions liées à la fragmentation, au déplacement et à la muséalisation des objets immobiliers apparentés à l'art brut, voir Patricia Allio, L'art brut déplacé. Hommage à Jean Grard, Paris, Apogée, 2006.

42. Voir cat. d'exp., op. cit. note 40.

43. Depuis 1999, le LaM possède la plus importante collection française d'art brut issue de la donation L'Aracine. Voir Roberta Trapani, « II LaM : un museo per I'arte moderna, I'arte contemporanea e I'Art Brut », revue de I'Osservatorio Outsider Art, Université de Palerme, $\mathrm{n}^{\circ} 2$, mars 2011, pp. 173-185. http://outsiderart.unipa.it/images/stories/pdf/Rivista/ooa_N2.pdf 44. Savine Faupin, « Habiter poétiquement », séminaire du CrAB Architectures hors discipline. Émergence, institutionnalisation et perspectives de conservation d'une pratique en voie d'artification, organisée par Roberta Trapani avec la collaboration d'Émilie Champenois, INHA, 24 novembre 2012. Actes à paraître.

45. B. Lassus, op. cit. note 6. 
associées à des images d'archives et à d'autres fragments, permettent de recomposer en pensée le site éclaté du sculpteur belge. Cette restitution est proposée en 2002 lors de l'exposition Les Chemins de l'art brut (1 $)^{46}$, puis, dès 2010, dans une salle du musée spécifiquement aménagée pour les habitants-paysagistes.

Si la conservation des objets immobiliers apparentés à l'art brut soulève toujours de profondes considérations esthétiques, pragmatiques et économiques qui peuvent paraître parfois insolubles, l'ensemble d'opérations mené par le LaM fournit une réponse à la question du morcellement des sites. Dans cette opération de reconstruction sur la base d'un archivage, les différents types de collecte - de photographies, de fragments, de données - abordés dans cet écrit s'associent, en renouant le fil du parcours en société de ces objets, un parcours sinueux marqué par le changement.

\section{L'auteur}

Après avoir obtenu une maîtrise en Histoire de l'Art à l'Université de Palerme, Roberta Trapani a poursuivi sa formation à l'Université Paris-Ouest Nanterre, où elle a orienté ses recherches sur l'œuvre environnementale de Niki de Saint Phalle, et notamment sur le Jardin des Tarots. Elle prépare actuellement une thèse d'histoire de l'art co-dirigée par Fabrice Flahutez et Eva di Stefano, à l'Université Paris-Ouest Nanterre La Défense et à l'Université de Palerme. Ses recherches portent sur la question des architectures sans architecte avec un fort ancrage vingtièmiste, mais posent aussi la question de ces objets immobiliers en rapport avec la création artistique et les pratiques marginales liées à l'art brut. Elle enseigne l'histoire de l'art contemporain à l'UCP de Paris-Ouest, est membre du $\mathrm{CrAB}$ (Collectif de réflexion autour de l'art brut) et de l'Osservatorio Outsider Art (Université de Palerme).

46 Les Chemins de l'art brut (1), cat. d'exp., sous la direction de Joëlle Pijaudier-Cabot et Christophe Boulanger, Musée d'art moderne Lille Métropole, 1 juin-2 septembre 2002, Musée d'art moderne Lille Métropole, Villeneuve d'Ascq, 2002, pp. 54-62. Voir aussi Habiter poétiquement le monde, cat. d'exp., sous la direction de Christophe Boulanger, Savine Faupin, François Piron, LaM, 25 septembre-30 janvier 2011, Liège, éd. LaM, 2010. 